\title{
Local Outbreak of COVID-19 in Shunyi District Attributed to an Asymptomatic Carrier with a History of Stay in Indonesia - Beijing Municipality, China, December 23, 2020
}

\author{
COVID-19 Epidemiology Investigation Team ${ }^{1}$; Laboratory Testing Team ${ }^{1}$; Wenzeng Zhang ${ }^{1, \#}$
}

\section{Summary}

What is known about this topic?

Patients with coronavirus disease 2019 (COVID-19) infection can be categorized by severity: asymptomatic infection, mild illness, moderate illness, severe illness, and critical illness. The rate of transmission to a specific group of contacts (the secondary attack rate) may be 3-25 times lower from people who are asymptomatically infected than from those with symptoms. The incubation period is $2-14$ days.

What is added by this report?

An individual with asymptomatic infection shed live virus that started a 42-case outbreak in Shunyi District of Beijing in December 2020. The individual had been quarantined for 14 days in a designated quarantine hotel in Fuzhou after entering China from Indonesia. During quarantine, he had 5 negative throat swab tests and 2 negative IgM serum tests. The investigation team determined that the incubation periods of 31 confirmed cases ranged from 2 to 23 days.

What are the implications for public health practice?

The frequency of nucleic acid testing should be increased for international entrants and people who are close contacts of confirmed COVID-19 cases. The investigation team recommend expanding sample types for nucleic acid testing to include anal swabs and residential environmental sampling; serum antibody testing can be used as a reference indicator. Parallel testing by two institutions at key points in time, such as the end of isolation, should be implemented.

As of December 22, 2020, no local cases of coronavirus disease 2019 (COVID-19) had been reported from Shunyi District of Beijing for 319 days. On December 23, the Shunyi District CDC received a report of a confirmed COVID-19 case, and another case was reported 2 days later. By January 31, 2021, 42 cases had been reported in Shunyi - a confirmed, local COVID-19 outbreak.
Shunyi CDC immediately launched an epidemiological investigation with laboratory testing to identify the source of infection, determine routes of transmission, assess the scale of the outbreak, and provide recommendations for stopping the outbreak and preventing recurrence. The investigation showed that all confirmed COVID-19 cases were associated with an asymptomatic carrier who was an international traveler from Indonesia. The investigation serves as a reminder that the government should pay attention to asymptomatic infections in our COVID-19 prevention and control strategies, including international entrant screening policies and practices.

\section{INVESTIGATION AND RESULTS}

At 05:08 on December 23, 2020, the index case of this local outbreak (Patient A) was reported to Shunyi CDC as a confirmed COVID-19 infection. Shunyi $\mathrm{CDC}$ conducted in-person interviews with a detailed questionnaire that used China CDC definitions for suspected and confirmed COVID-19 cases (1-2). A series of response measures were implemented but contact tracing did not immediately lead to source identification.

At $05: 30$ on December 25, 2 days later, a second case (Patient B) was reported to Shunyi CDC. Patient $B$ had nausea, sore throat, and dizziness at 18:00 on December 16, but did not seek medical attention at that time. An investigation found that Patient B had a roommate who was a 28 -year old Indonesian man who had returned from Indonesia on November 26. The person from Indonesia had entered China at Fuzhou Airport; en route, he sat in seat 60L; a nearby passenger in seat 60J was later confirmed as a COVID-19 case. Because he was a close contact of a confirmed case, the person from Indonesia was required to be in medical quarantine from November 26 to December 10 . During quarantine, five consecutive COVID-19 nucleic acid tests and two consecutive virus-specific 
antibodies tests were performed; all tests were negative.

After completing the 14-day quarantine, the individual from Indonesia travelled from Fuzhou to Beijing, arriving at 00:05 on December 11. He rented an apartment that day in a community in Shunyi District through a housing agency; there were four others in the apartment. One of the other four was Patient B.

From December 12 to 20, the person from Indonesia was required to conduct quarantine at the apartment, but he did not strictly abide by the quarantine regulations. During this period, he went to malls, supermarkets, and restaurants. At 15:17 on December 13, he went to Hualian Mall and returned to the rental unit at 16:51. Two people with whom he had close contact in Hualian Shopping Center became Patient C (a salesperson in the mall) and Patient D. Further investigations revealed that Patient $\mathrm{A}$ had close contact with Patient $\mathrm{C}$ while at Hualian Shopping Center on December 17.

From December 21 to 24, the person from Indonesia took the subway to work at a company in Wangjing, Chaoyang District around 09:00 every day and took the subway back to the apartment after getting off work around 19:00, arriving home around 20:00. He stated that he was wearing a disposable mask during shopping and work. Between December 10 and 24, no throat swabs or serum specimens from this person were obtained.

At $09: 28$ on December 25, the person from Indonesia was determined to be a close contact of Patient B and was transferred to a hotel designated for medical quarantine at $15: 14$. Throat swabs and serum specimens were collected and tested for viral nucleic acids and virus-specific antibodies respectively. Sera tested positive for IgM and negative for IgG specific to the COVID-19 virus.

At $14: 30$ on December 27, the person from Indonesia was transferred to Beijing Ditan Hospital for further diagnosis and treatment. On December 28, his throat swab and stool specimens were positive for COVID-19, with cycle threshold (Ct) values of 39.0/37.0 (ORF1ab/N), and 24-28/24-28. At 22:00, he was diagnosed as an asymptomatic COVID-19 carrier (Patient E).

Between December 10, 2020 and January 31, 2021, 42 COVID-19 cases were identified and linked to this outbreak. Starting on December 25, the number of cases significantly increased, reaching a peak on December 27 and 28 (Figure 1).

The 42 cases involved 5 generations of infection - 1 case in the first generation (Patient E), 3 in the second generation, 13 in the third generation, 22 in the fourth generation, and 3 in the fifth generation. The secondgeneration cases were infected when living together or during close contact in a shopping mall with Patient E. Among the second-generation cases, one resided with Patient $\mathrm{E}$ and the other two were close contacts of Patient E in the Hualian Mall on December 13. The third-generation cases were infected when living together or traveling in cars, or when in close contact with second-generation cases in the mall. The fourth and fifth generation cases were infected when eating or working with cases.

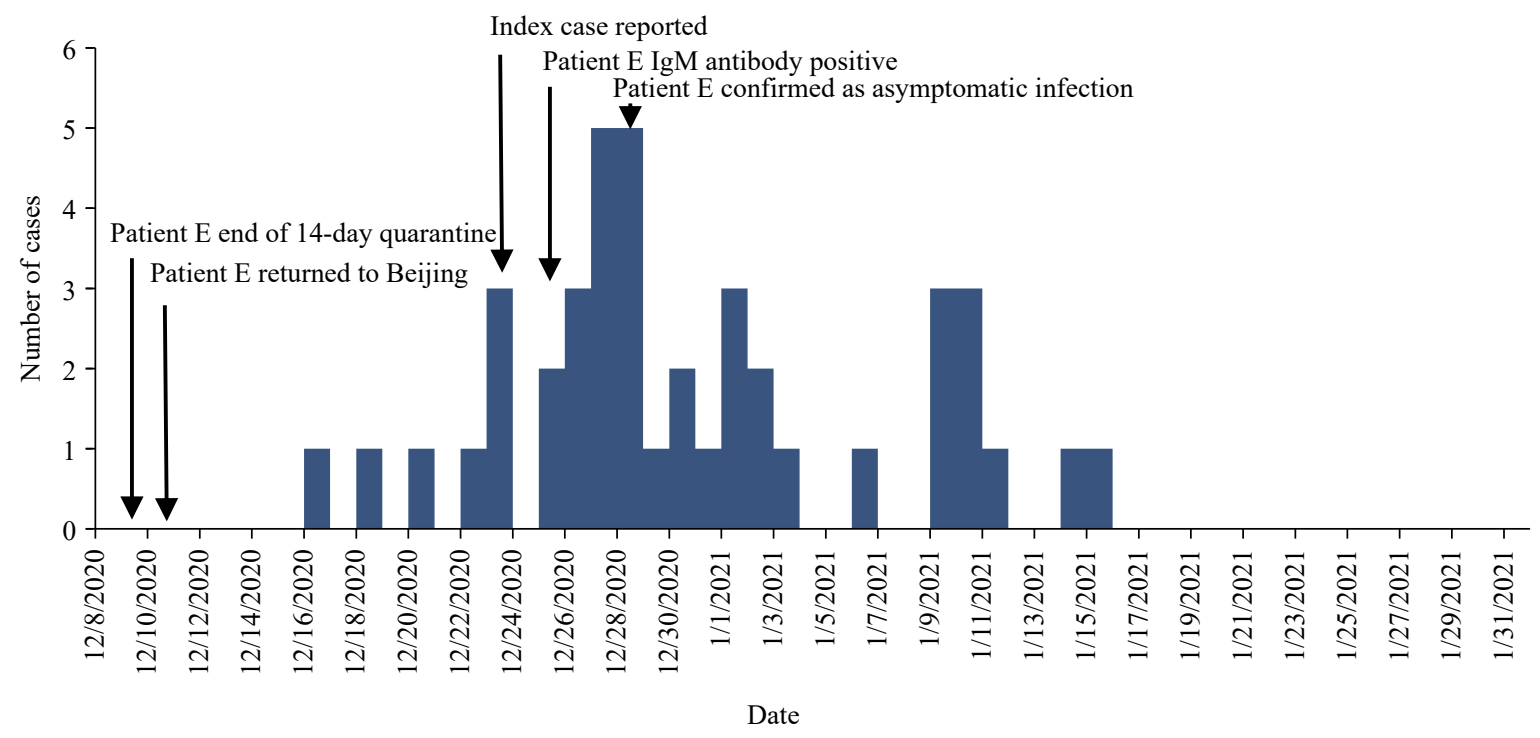

FIGURE 1. Epidemiological curve of a local outbreak of COVID-19 in Shunyi District, Beijing, 2020-2021. 
According to our epidemiological investigation, 31 of the 42 outbreak cases had clear last exposure times. Based on data from these 31 cases, we calculated the median incubation period to be 5 days, with a range of 2 to 23 days.

In total, 41 cases were reported from Shunyi District, and 1 was reported from another district in Beijing. The median age was 32 years $(8$ months -62 years); the male:female ratio was $1.6: 1 ; 18(42.9 \%)$ were workers, and $8(19 \%)$ were unemployed. There were no severe or critically ill cases. Overall, 24 cases (57.1\%) were moderate illness, $10(23.3 \%)$ were mild illness, and $8(18.2 \%)$ were asymptomatic. Except Patient E, the remaining 7 cases of asymptomatic infection were confirmed by the test results of throat swabs or anal swabs for COVID-19 (Table 1).

The investigation for Patient E obtained 203 environmental specimens during December 26 and 27, including 21 samples from the apartment, among which 9 tested positive for COVID-19 virus, and 183 samples from the workplace, among which 5 were positive and 1 was indeterminate.

Full-length genome sequences were determined for 14 specimens from people, including Patient E, and 5 environmental samples ( 2 were from the apartment and the workplace of Patient E). Sequence alignment and phylogenetic analyses showed that all 19 strains were highly homologous, with similarities over 99.993\% and having only 1-2 sites with nucleotide variations. The strain that caused this local outbreak of COVID-19 in Shunyi District belonged to the LLineage European Branch 2.3 (B.1.160.3) and has high homology with COVID-19 strains found in Southeast Asia during the same period.

Ultimately, Shunyi CDC identified asymptomatic
Patient $\mathrm{E}$ as the source of infection for this outbreak in Shunyi District.

\section{PUBLIC HEALTH RESPONSE}

Shunyi CDC conducted face-to-face interviews with all confirmed cases to ascertain and record their activities during the 14 days before onset of symptoms or positive nucleic acid tests. Contact times (exposure times) between cases were evaluated and incubation were calculated. Close contacts had been advised to quarantine themselves at designated hotels for 21 days and at home for 7 days, with daily check-in by medical staff and body temperature readings twice daily. Contacts developing fever or respiratory symptoms were transferred immediately to designated medical institutions for diagnosis and treatment, if needed. Throat swabs, anal swabs, and serum specimens were obtained from all subjects, close contacts, and other key individuals. Environmental samples from the patient's residence, workplace, and points visited during the four days before illness onset or positive nucleic acid testing were obtained and tested (3). Parallel testing of samples by two institutions at key time points were implemented. Social distancing measures, discouragement of mass gatherings, mass testing of the entire population, closed management of the community where the COVID-19 cases were located, and health code scanning and registration of people entering public places had also been strictly implemented.

\section{DISCUSSION}

The results of this outbreak investigation remind us

TABLE 1. The first sampling and testing results of 8 cases of asymptomatic infection of a local outbreak of COVID-19 in Shunyi District, Beijing, 2020-2021.

\begin{tabular}{|c|c|c|c|c|c|c|}
\hline \multirow{2}{*}{ Patient } & \multicolumn{2}{|c|}{ Throat swab } & \multicolumn{2}{|c|}{ Anal swab } & \multicolumn{2}{|c|}{ Serum specimen } \\
\hline & ORF 1a/b (A lab/B lab) & N (A lab/B lab) & ORF 1a/b (A lab/B lab) & N (A lab/B lab) & $\lg M$ & $\lg G$ \\
\hline$E$ & $-1-$ & $-1-$ & $-1-$ & $-1-$ & + & - \\
\hline $\mathrm{F}$ & $+1+$ & $+1+$ & $-1-$ & $-1-$ & - & - \\
\hline G & $+/ \mathrm{n} . \mathrm{a}$ & $+/$ n.a. & - In.a. & - /n.a. & - & - \\
\hline $\mathrm{H}$ & $+1+$ & $+1+$ & $+1+$ & $+1+$ & - & - \\
\hline I & $+1+$ & $+1+$ & $-1-$ & $-1-$ & - & - \\
\hline $\mathrm{J}$ & $-1-$ & $-1-$ & $+1+$ & $+1+$ & - & - \\
\hline $\mathrm{K}$ & $+1+$ & $+1+$ & $+1+$ & $+1+$ & - & - \\
\hline L & $+1-$ & $-1+$ & $-1-$ & $+1-$ & - & - \\
\hline
\end{tabular}

Abbreviation: ORF $1 \mathrm{a} / \mathrm{b}=$ Open reading frame $1 \mathrm{a} / \mathrm{b} ;$ n.a.=not applicable. 
that we must pay attention to asymptomatically infected patients for the prevention and control of COVID-19. If an asymptomatic infected individual is not detected in time, he or she can cause a serious public health event (4-6). Early detection, early reporting, early isolation, and early treatment of asymptomatic patients require a joint effort with policymakers, clinicians, technicians, epidemiologists, virologists, and patients.

The results of this outbreak investigation showed that Patient E went to Shunyi District, Beijing after the 14-day quarantine that is required of international entrants to China. During the next seven days, Patient E went shopping and dining many times and was not strictly quarantining himself at home. Local staff did not manage this individual properly as an international entrant to China. As a result, he had contact with four co-residents including Patient B. On the Day 18 after entry, he had short-term, close contact in the mall with patients $\mathrm{C}$ and D. Patients B, C, and D later became confirmed COVID-19 cases. The turn of events revealed gaps in the management of international entrants and showed that the community supervision responsibility had not been thoroughly implemented. We recommend that for international entrants and people in close contact with COVID-19 cases after the 14-day intensive medical quarantine, at least 7 days of home quarantine are necessary. Local staff should tightly implement their responsibilities for infectious disease management and strictly manage home quarantine.

Patient E was asymptomatic and was not detected and isolated in time, enabling virus transmission that started this local COVID-19 outbreak. Unnoticed, asymptomatically infected individuals may accelerate person to person spread of COVID-19. Therefore, effective screening and management of asymptomatic but infected individuals is a key requirement for successfully controlling virus spread in the COVID-19 pandemic. For international entrants and people who are in close contact with COVID-19 cases, the frequency of nucleic acid testing should be increased. We propose expanding nucleic acid testing and screening and adding anal swabs and residence environmental specimens to the set of tests to perform; serum antibody testing can also be used as a reference indicator. Parallel testing by two institutions at key management time points should be implemented.

Acknowledgement: Lijie Zhang of the Chinese Field Epidemiology Training Program of China CDC.

Field teams on case and close contact tracing: Beijing Municipality CDC Team; Daxing District CDC Team; Fengtai District CDC Team; Chaoyang District CDC Team; Fangshan District CDC Team; Mentougou District CDC Team; Chaoyang District CDC Team.

doi: $10.46234 / \mathrm{ccdcw} 2020.062$

\# Corresponding author: Wenzeng Zhang, zeng4379@126.com. ${ }^{1}$ Shunyi District Center for Disease Control and Prevention, Beijing,
China.

Submitted: February 10, 2021; Accepted: February 22, 2021

\section{REFERENCES}

1. Chinese Center for Disease Control and Prevention. Guidelines for COVID-19 epidemiological investigations. China CDC Wkly 2020;2(19):327 - 8. http://dx.doi.org/10.46234/ccdcw2020.083.

2. Wang P, Lian ZY, Chen Y, Qi Y, Chen HJ, An XD. Investigation of a cluster of 2019 Novel Coronavirus Disease (COVID-19) with possible transmission during the incubation period-Shenyang City, China, 2020. China CDC Wkly 2020;2(8):125-7. http://dx.doi.org/10. 46234/ccdcw2020.034.

3. Chinese Center for Disease Control and Prevention. Technical guidelines for COVID-19 laboratory testing. China CDC Wkly 2020;2(19):332 6. http://dx.doi.org/10.46234/ccdcw2020.085.

4. Rivett L, Sridhar S, Sparkes D, Routledge M, Jones NK, Forrest F, et al. Screening of healthcare workers for SARS-CoV-2 highlights the role of asymptomatic carriage in COVID-19 transmission. eLife 2020;9:e58728. http://dx.doi.org/10.7554/eLife.58728.

5. Yu XX, Yang RR. COVID-19 transmission through asymptomatic carriers is a challenge to containment. Influenza Other Respir Viruses 2020;14(4):474 - 5. http://dx.doi.org/10.1111/irv.12743.

6. Han DS, Li R, Han YX, Zhang R, Li JM. COVID-19: insight into the asymptomatic SARS-COV-2 infection and transmission. Int J Biol Sci 2020;16(15):2803 - 11. http://dx.doi.org/10.7150/ijbs.48991. 